\title{
FIXED POINT THEOREMS USING (CLCS) PROPERTY IN COMPLEX VALUED b-METRIC SPACES
}

\author{
Rohit Kumar Verma
}

\begin{abstract}
Various common fixed point theorems have been proved for one or two pairs of mappings using either $(C L R)$ property $([44])$, or by taking one of the range-subspace closed. In this paper, we introduce the notion of (CLCS)-property i.e., "common limit converging in the range sub-space". Using this property, we prove common fixed point theorems for two pairs of weakly compatible mappings in complex valued b-metric spaces satisfying a collection of contractive conditions. Our notion is meaningful and valid because the required common fixed point will always lie on the range-subspace of the mapping-pair. We give some examples to show that if a mapping pair $(f, g)$ on a closed complex valued b-metric space $X$ satisfy the $\left(C L R_{f}\right)$ property, then it is also $\left(C L R_{g}\right)$, and vice-versa.

Keywords: Banach contraction principal, common fixed point, complete metric space, complex valued metric space, complex valued $b$-metric space, weakly compatible mappings
\end{abstract}

\section{Introduction}

Banach contraction principal [7] is a fundamental result in fixed point theory. This theorem has been generalized in many ways. Bakhtin [8] introduced the notion of $b$-metric space as a generalization of metric space in which the triangle inequality is relaxed. Further, Czerwik [13] proved a contraction theorem in this space, which generalized the Banach contraction principal. Malhotra and Bansal [28] proved some common coupled fixed point theorems for generalized contraction in $b$-metric spaces.

Azam et al. [4] introduced the complex valued metric space which is a generalization of the metric space. They obtained some fixed point results for a pair of mappings satisfying a rational inequality. Further, Bhatt et al. [5], [6] generalized the result of Azam et al. [4]. In this line, Ahmad et al. [2], Chandok and Kumar [9], Hakwadia et al. [15], Manro [24] Öztürk [29], Öztürk and Kaplan [30],

Received March 16, 2015; accepted December 11, 2015

2010 Mathematics Subject Classification. Primary 47H10; Secondary 54H25 
Sharma [41] and Sitthikul and Saejung [48] etc. proved some common fixed point results for mappings satisfying contractive condition in complex valued metric spaces.

Recently, Mukheimer [27], Rao et al. [35], Dubey et al. [14] and Singh et al. ([46], [47]) etc. proved some common fixed point results in complex valued $b$-metric spaces.

On the other hand, Jungck [19] introduced the notion of compatible mappings, which was defined for a sequence in the metric space $X$. Along this line, Pant [31] proved some common fixed point results using non-compatible mappings. The noncompatibility was further generalized to property (E.A) by Aamri and Moutawakil [3]. Pathak, Lopez and Verma ([32], [34]) gave various examples on weak compatibility and property (E.A) in a metric space satisfying an implicit relation. Verma and Pathak [50] also proved a fixed point result using property (E.A) in a complex valued metric space. Further, Sintunavarat and Kumam [44] introduced the concept of (CLR)-property (i.e., common limit in the range of a mappings). Many fixed point theorems have been proved using this property, e.g., [44], [45], [48] etc.

In the line of 'common limit range' property, Chouhan et al. [10] introduced the (JCLR) property. Further, Imdad et al. [16] used this property for two hybrid pairs of non-self mappings and utilized the same to obtain some coincidence and common fixed point theorems defined on an arbitrary set with values in metric spaces.

Similarly, Chauhan, Khan and Kumar, [12] proved a unified common fixed point theorem (Theorem 4.1 with the help of Lemma 3) via common limit range property in fuzzy metric spaces, for two pairs of weakly compatible mappings satisfying an implicit relation defined on a set of all continuous functions $\phi:[0,1]^{6} \rightarrow \mathbb{R}$. However, Theorem 4 and Lemma 3, is found incorrect by Rolden, Karapinar and Kumam [37]. They improved and generalized this theorem and lemma (Ref. Theorem 11 of [37]) by proposing Axiom (FM-6) in fuzzy metric space.

Pathak et. al. [33] introduced the concept of R-weakly commuting of type $\left(A_{g}\right)$ in a metric space. Sintunavarat and Kumam [43] used this concept in a fuzzy metric space and established a common fixed point theorem by using the common limit in the range property.

X. Q. Hu [51] proved common coupled fixed point theorems for contractive mappings in a fuzzy metric spaces. Jain et al. [18] extend the notion of (EA) and $\left(C L R_{g}\right)$ properties for coupled mappings and generalized the result of X. Q. Hu [51].

We will show below, by an example, that if a mapping pair $(f, g)$ of a space $X$ satisfy the $\left(C L R_{f}\right)$ property, then under some conditions it is $\left(C L R_{g}\right)$, and viceversa. Thus we may unify the $\left(C L R_{f}\right)$ and $\left(C L R_{g}\right)$ properties for these conditions. We hint here that the common fixed point will always lie on the intersection of 
the range-subset of the mapping pair. We will call this a (CLCS)-property, (or, "common limit converging in the range sub-space"). The (CLCS)-property unifies both $\left(C L R_{f}\right)$ and $\left(C L R_{g}\right)$ properties, in which the fixed point will necessarily lie, if it exist.

Furthermore, in 2014, Ahmad et. al [2] obtained a common fixed point result for a pair of mappings satisfying rational expressions on a closed ball in a complex valued metric space. In this paper, we will unify the $\left(C L R_{f}\right)$ and $\left(C L R_{g}\right)$ properties, which is defined in the range subspace $f(X) \bigcap g(X)$. If this is a closed subset, then we need not to take the closed ball, unlike [2], in which the contractive condition satisfy.

A further generalization of compatible mappings, namely weakly compatible mappings, was introduced by Jungck [20]. More results on complex valued metric spaces using weak compatibility can be found in [9], [21], [23], [26], [29], [30], [39], [40], [41], [44], [45], [48] and [50] etc.

In this paper, we introduce the notion of (CLCS)-property and prove a common fixed point theorem for two pairs of weakly compatible mappings in complex valued b-metric spaces satisfying a collection of contractive conditions.

\section{Preliminaries}

Definition 2.1. ([28]) Let $X$ be a nonempty set and let $s \geq 1$ be a real number. The mapping $d: X \times X \rightarrow[0, \infty)$ is called b-metric space if for all $x, y, z \in X$ :

(B1) $\quad 0 \leq d(x, y)$ and $d(x, y)=0$ if and only if $x=y$,

(B2) $\quad d(x, y)=d(y, x)$,

(B3) $d(x, y) \leq s[d(x, z)+d(z, y)]$,

satisfy. The number $s$ is called the coefficient or parameter of the b-metric space.

Example 2.1. ([1], [28]) Let $(X, d)$ be a metric space and $\rho(x, y)=(d(x, y))^{p}$, where $p>1$ is a real number. Then $(X, \rho)$ is a b-metric space with $s=2^{p-1}$.

Every b-metric is a metric, but there may exist b-metric which is not a metric, as shown in the following example:

Example 2.2. ([1], [36]) If $X=\mathbb{R}$ is the set of real numbers and $d(x, y)=|x-y|$ is the usual Euclidean metric, then $\rho(x, y)=(d(x, y))^{2}=|x-y|^{2}$ is a b-metric on $\mathbb{R}$ with $s=2$; but is not a metric on $\mathbb{R}$ (see Ex.1.1 of [36]).

Let $\mathbb{C}$ is the set of complex numbers $z=a+i b$. Here $a, b$, are real numbers, $a$ is called $\operatorname{Re}(z)$ and $b$ is called $\operatorname{Im}(z)$. A complex valued metric $d$ is a function from a set $X \times X$ into $\mathbb{C}$. Let $z_{1}, z_{2} \in \mathbb{C}$; define a partial order $\precsim$ on $\mathbb{C}$ as follows:

$$
z_{1} \precsim z_{2} \text { ifandonlyif } \operatorname{Re}\left(z_{1}\right) \leq \operatorname{Re}\left(z_{2}\right), \operatorname{Im}\left(z_{1}\right) \leq \operatorname{Im}\left(z_{2}\right) .
$$


It follows that $z_{1} \precsim z_{2}$ if one of the following conditions is satisfied:

(i) $\operatorname{Re}\left(z_{1}\right)=\operatorname{Re}\left(z_{2}\right), \operatorname{Im}\left(z_{1}\right)<\operatorname{Im}\left(z_{2}\right)$,

(ii) $\operatorname{Re}\left(z_{1}\right)<\operatorname{Re}\left(z_{2}\right), \quad \operatorname{Im}\left(z_{1}=\operatorname{Im}\left(z_{2}\right)\right.$,

(iii) $\operatorname{Re}\left(z_{1}\right)<\operatorname{Re}\left(z_{2}\right), \operatorname{Im}\left(z_{1}\right)<\operatorname{Im}\left(z_{2}\right)$,

(iv) $\operatorname{Re}\left(z_{1}\right)=\operatorname{Re}\left(z_{2}\right), \quad \operatorname{Im}\left(z_{1}\right)=\operatorname{Im}\left(z_{2}\right)$.

In (i), (ii) and (iii), we have $\left|z_{1}\right|<\left|z_{2}\right|$. In (iv), we have $\left|z_{1}\right|=\left|z_{2}\right|$. So $\left|z_{1}\right| \leq\left|z_{2}\right|$, whenever $z_{1} \precsim z_{2}$. In particular, $z_{1} \precsim z_{2}$ if $z_{1} \neq z_{2}$ and one of (i), (ii), (iii) satisfy. In this case $\left|z_{1}\right|<\left|z_{2}\right|$. We will write $z_{1} \prec z_{2}$ if only (iii) satisfy. Hence $z_{1} \prec z_{2} \Rightarrow\left|z_{1}\right|<\left|z_{2}\right|$.

Remark 2.1. ([21]) We note that the following statements hold:

(i) $a, b \in \mathbb{R}$ and $a \leq b \Rightarrow a z \precsim b z, \forall z \in \mathbb{C}$;

(ii) $0 \precsim z_{1} \precsim z_{2} \Rightarrow\left|z_{1}\right|<\left|z_{2}\right|, \forall z_{1}, z_{2} \in \mathbb{C}$;

(iii) $z_{1} \precsim z_{2}$ and $z_{2} \prec z_{3} \Rightarrow z_{1} \prec z_{3}, \forall z_{1}, z_{2}, z_{3} \in \mathbb{C}$.

Azam et al. [4] defined complex-valued metric space $(X, d)$ in the following way:

Definition 2.2. [4] Let $X$ be a nonempty set. Suppose that the mapping $d$ : $X \times X \rightarrow \mathbb{C}$ satisfies the following conditions:

(A1) $\quad 0 \precsim d(x, y)$, and $d(x, y)=0$ if and only if $x=y, \forall x, y \in X$,

(A2) $\quad d(x, y)=d(y, x), \forall x, y \in X$,

(A3) $\quad d(x, y) \precsim d(x, z)+d(z, y), \forall x, y, z \in X$,

then $d$ is called complex-valued metric, and $(X, d)$ is called a complex-valued metric space.

On generalizing (A3) above, i.e., the triangle inequality, in the following way, complex valued b-metric space is defined:

Definition 2.3. ([27], [28], [35]) Let $X$ be a nonempty set. Suppose that the mapping $d: X \times X \rightarrow \mathbb{C}$ satisfy the following conditions:

(C1) $\quad 0 \precsim d(x, y)$, and $d(x, y)=0$ if and only if $x=y$, for all $x, y \in X$,

(C2) $\quad d(x, y)=d(y, x)$ for all $x, y \in X$,

(C3) $d(x, y) \precsim s[d(x, z)+d(z, y)], \forall x, y, z \in X$, where $s \geq 1$ is a real number, then $d$ is called a complex valued $b$-metric on $X$, and $(X, d)$ is called complex valued b-metric space.

Example 2.3. Let $X=\mathbb{C}$ and $d: X \times X \rightarrow \mathbb{C}$ be defined by: $d\left(z_{1}, z_{2}\right)=5 i\left|z_{1}-z_{2}\right|^{2}$. To show that $(X, d)$ is a complex valued b-metric space with $s=2$, it is enough to verify the triangular inequality condition. For, let $z_{1}, z_{2}, z_{3} \in \mathbb{C}$, then

$$
\begin{aligned}
d\left(z_{1}, z_{3}\right)= & 5 i\left|z_{1}-z_{3}\right|^{2}=5 i\left(\left|\left(z_{1}-z_{2}\right)+\left(z_{2}-z_{3}\right)\right|\right)^{2} \precsim 5 i\left(\left|z_{1}-z_{2}\right|+\left|z_{2}-z_{3}\right|\right)^{2} \\
& \precsim 5 i\left(\left|z_{1}-z_{2}\right|^{2}+\left|z_{2}-z_{3}\right|^{2}+2\left|z_{1}-z_{2}\right| \cdot\left|z_{2}-z_{3}\right|\right) \\
& \precsim 5 i\left(\left|z_{1}-z_{2}\right|^{2}+\left|z_{2}-z_{3}\right|^{2}+2 \cdot \frac{1}{2}\left\{\left|z_{1}-z_{2}\right|^{2}+\left|z_{2}-z_{3}\right|^{2}\right\}\right), \quad(\mathrm{AM} \geq \mathrm{GM}) \\
& =5 i\left(2\left|z_{1}-z_{2}\right|^{2}+2\left|z_{2}-z_{3}\right|^{2}\right)=2\left(d\left(z_{1}, z_{2}\right)+d\left(z_{2}, z_{3}\right)\right) .
\end{aligned}
$$

Thus $(X, d)$ is a complex valued b-metric space with $s=2$. 
Example 2.4. ([35]) Let $X=[0,1]$. Define a complex valued metric $d: X \times X \rightarrow \mathbb{C}$ by: $d(x, y)=|x-y|^{2}+i|x-y|^{2}, \forall x, y \in X$; then $(X, d)$ is a complex valued b-metric space with $\mathbf{s}=\mathbf{2}$.

Remark 2.2. Note that, if $s=1$ then the complex valued $b$-metric space reduces to a complex valued metric space. Thus every complex valued metric space is a complex valued b-metric space, but not conversely. This generalizes the concept of a complex valued $b$-metric space over the complex valued metric space.

Definition 2.4. ([35]) Let $(X, d)$ be a complex valued b-metric space, then

(1) A point $x \in X$ is called interior point of a set $A \subseteq X$ whenever $\exists 0 \prec r \in \mathbb{C}$ : $B(x, r)=\{y \in X: d(x, y) \prec r\} \subseteq A$.

(2) A point $x \in X$ is called the limit point of a set $A \subseteq X$ whenever for every $0 \prec r \in \mathbb{C}: B(x, r) \bigcap(X-A) \neq \phi$.

(3) A subset $B \subseteq X$ is called open whenever each element of $B$ is an interior point of $B$.

(4) A subset $B \subseteq X$ is called closed whenever each limit point of $B$ belongs to $B$.

(5) The family $\mathcal{F}:=\{B(x, r): x \in X, 0 \prec r\}$ is a sub-basis for a topology on $X$. We denote this complex topology by $\tau_{C}$. Indeed the topology $\tau_{C}$ is Hausdorff.

Definition 2.5. [35] Let $(X, d)$ be a complex valued b-metric space, and let $\left\{x_{n}\right\}$ be a sequence in $X$ and $x \in X$.

(1) If for every $c \in \mathbb{C}$ with $0 \prec c \exists n_{0} \in \mathbb{N}: \forall n>n_{0}, d\left(x_{n}, x\right) \prec c$, then $\left\{x_{n}\right\}$ is said to be convergent to $x$, and $x$ is limit point of $\left\{x_{n}\right\}$. We denote this by $x_{n} \rightarrow x$ as $n \rightarrow \infty$, or $\lim _{n \rightarrow \infty} x_{n}=x$.

(2) If for every $c \in \mathbb{C}$ with $0 \prec c$ there is $n_{0} \in \mathbb{N}: \forall n>n_{0}, d\left(x_{n}, x_{m}\right) \prec c$, where $m \in \mathbb{N}$, then $\left\{x_{n}\right\}$ is said to be Cauchy sequence.

(3) If every Cauchy sequence of $X$ converges in $X$, then $X$ is called the complete complex valued b-metric space.

Definition 2.6. [19] Let $f, g: X \rightarrow X$. Suppose $\left\{x_{n}\right\}$ be a sequence in $X$ such that $\lim _{n \rightarrow \infty} f x_{n}=\lim _{n \rightarrow \infty} g x_{n}=t$, for some $t \in X$. Then $f, g: X \rightarrow X$ is called compatible mappings if $\lim _{n \rightarrow \infty} d\left(f g x_{n}, g f x_{n}\right)=0$.

Definition 2.7. [31] Let $f, g: X \rightarrow X$. Suppose $\left\{x_{n}\right\}$ be a sequence in $X$ such that $\lim _{n \rightarrow \infty} f x_{n}=\lim _{n \rightarrow \infty} g x_{n}=t$, for some $t \in X$. Then $f, g: X \rightarrow X$ is called noncompatible mappings if the $\operatorname{limit}_{\lim _{n \rightarrow \infty}} d\left(f g x_{n}, g f x_{n}\right)$ is either nonzero, or nonexistent.

Definition 2.8. [3] Let $f, g: X \rightarrow X$. Then pair $(f, g)$ is said to satisfy property (E.A), if there exist a sequence $\left\{x_{n}\right\}$ in $X$ such that $\lim _{n \rightarrow \infty} f x_{n}=\lim _{n \rightarrow \infty} g x_{n}=$ $t$, for some $t \in X$.

Definition 2.9. [44] Let $f, g: X \rightarrow X$. Suppose $\left\{x_{n}\right\}$ be a sequence in $X$. Then the pair $(f, g)$ is said to satisfy property $\left(C L R_{f}\right)$, if $\lim _{n \rightarrow \infty} f x_{n}=\lim _{n \rightarrow \infty} g x_{n}=$ 
$f u$, for some $u \in X$. Similarly, the pair $(f, g)$ is said to satisfy property $\left(C L R_{g}\right)$, if $\lim _{n \rightarrow \infty} f x_{n}=\lim _{n \rightarrow \infty} g x_{n}=g v$, for some $v \in X$. The "common limit in the range of $f$ " is called $\left(C L R_{f}\right)$ and the "common limit in the range of $g$ " is called $\left(C L R_{g}\right)$ property, respectively.

See Ex. 2.4 for CLR (common limit in the range) property, and Ex. 2.5 for JCLR (jointly common limit in the range) property in Manro [24].

Remark 2.3. Let $(X, d)$ be a closed, complex valued b-metric space. Define $f, g: X \rightarrow$ $X$. Let $\left\{x_{n}\right\} \subseteq X$ be a sequence such that $\lim _{n \rightarrow \infty} f x_{n}=\lim _{n \rightarrow \infty} g x_{n}=g u=t$ (say). Then $(f, g)$ satisfy the $(E . A)$ property $([3])$ and $\left(C L R_{g}\right)$ property at $u([44]-[45])$. Since $X$ is closed so there exist some $u \in X$ such that $t=f u$. In this case, $(f, g)$ is $\left(C L R_{f}\right)$ at $u$. Thus $(f, g)$ satisfy both $\left(C L R_{f}\right)$ and $\left(C L R_{g}\right)$ properties.

Following example shows that under some conditions, if the pair of self-mappings $f, g: X \rightarrow X$ satisfy $\left(C L R_{f}\right)$-property, then it will also satisfy the $\left(C L R_{g}\right)$ property; and vice-versa. This will happen because the value of "common limit" (say $t=g v \in X$, in case of $\left(C L R_{g}\right)$-property); and the value of "common limit" (say $t=f u \in X$, in case of $\left(C L R_{f}\right)$ property) always belongs to $X$, for some $u, v$ necessarily belongs to $X$.

Example 2.5. Let $X=\mathbb{C}$ and $d$ be any complex valued metric on $X$. Define $f, g$ : $X \rightarrow X$ by: $f z=z+2 i, g z=3 z, \forall z \in X$. Consider a sequence $\left\{z_{n}\right\}=\left\{i+\frac{1}{n}\right\}$ in $X$, then $\lim _{n \rightarrow \infty} f z_{n}=\lim _{n \rightarrow \infty}\left(z_{n}+2 i\right)=\lim _{n \rightarrow \infty}\left(i+\frac{1}{n}\right)+2 i=3 i$, and $\lim _{n \rightarrow \infty} g z_{n}=$ $\lim _{n \rightarrow \infty} 3\left(i+\frac{1}{n}\right)=3 i=g(i)$. So that $\lim _{n \rightarrow \infty} f z_{n}=\lim _{n \rightarrow \infty} g z_{n}=g(i)$. Hence, the pair $(f, g)$ satisfy property $\left(C L R_{g}\right)$ with $v=i \in X$.

On the other hand, $\lim _{n \rightarrow \infty} f z_{n}=\lim _{n \rightarrow \infty} g z_{n}=3 i=f(i)$; hence the pair $(f, g)$ also satisfy property $\left(C L R_{f}\right)$ in $X$ with $u=i \in X$.

Example 2.6. Let $X=\mathbb{C}$ and $d\left(z_{1}, z_{2}\right)=(\cos \alpha+i \sin \alpha)\left|z_{1}-z_{2}\right|, \forall \alpha \in\left[0, \frac{\pi}{2}\right)$, be any complex valued metric on $X$. Define $f, g: X \rightarrow X$ by: $f z=2 z-4, g z=z+2 i, \forall z \in X$. Consider a sequence $\left\{z_{n}\right\}=\left\{4+2 i+\frac{1}{n}\right\}$ in $X$, then $\lim _{n \rightarrow \infty} f z_{n}=\lim _{n \rightarrow \infty} g z_{n}=(4+4 i)$. Observe that $4+4 i=f(4+2 i)$; hence, the pair $(f, g)$ satisfy $\left(C L R_{f}\right)$-property in $X$ with $u=4+2 i \in X$.

Further, observe that $4+4 i=g(4+2 i)$, hence $(f, g)$ satisfy $\left(C L R_{g}\right)$ property with $v=4+2 i \in X$. Thus if $(f, g)$ satisfy property $\left(C L R_{f}\right)$ then so is $\left(C L R_{g}\right)$.

Example 2.7. Let $X=\mathbb{C}$ and $d$ be any complex valued b-metric on $X$. Define $f, g: X \rightarrow X$ by: $f z=z^{2}-\frac{i}{3}, g z=-z+1, \forall z \in X$. Consider a sequence $\left\{z_{n}\right\} \subseteq X$; then for the "common limit" $t$ in the pair $(f, g)$, we must have

$$
\begin{aligned}
& \lim _{n \rightarrow \infty} f z_{n}=\lim _{n \rightarrow \infty} g z_{n} \quad \Leftrightarrow \quad \lim _{n \rightarrow \infty}\left(z_{n}^{2}-\frac{i}{3}\right)=\lim _{n \rightarrow \infty}\left(-z_{n}+1\right) \\
& \Leftrightarrow \lim _{n \rightarrow \infty}\left(z_{n}^{2}+z_{n}-1-\frac{i}{3}\right)=0 \quad \Leftrightarrow \quad \lim _{n \rightarrow \infty} z_{n}=\frac{-1}{2} \pm \frac{1}{2} \sqrt{\left(5+\frac{4 i}{3}\right)} .
\end{aligned}
$$

So, if we take a sequence $\left\{z_{n}\right\}_{n=1}^{n=\infty}:=\left\{\frac{1}{n}+\left(\frac{-1}{2}+\frac{1}{2} \sqrt{\left(5+\frac{4 i}{3}\right)}\right)\right\}_{n=1}^{n=\infty}$ then $\lim _{n \rightarrow \infty} f z_{n}=\lim _{n \rightarrow \infty}\left(z_{n}^{2}-\frac{i}{3}\right)=\lim _{n \rightarrow \infty}\left(1-z_{n}\right)=\lim _{n \rightarrow \infty}\left[1-\left\{\frac{1}{n}+\left(\frac{-1}{2}+\frac{1}{2} \sqrt{\left(5+\frac{4 i}{3}\right)}\right)\right\}\right]=$ $\frac{3}{2}-\frac{1}{2} \sqrt{\left(5+\frac{4 i}{3}\right)}=t=\lim _{n \rightarrow \infty} g z_{n}=g v=g\left(\frac{-1}{2}+\frac{1}{2} \sqrt{\left(5+\frac{4 i}{3}\right)}\right)$. 
Hence, the pair $(f, g)$ satisfy property $\left(C L R_{g}\right)$ with $v=\frac{-1}{2}+\frac{1}{2} \sqrt{\left(5+\frac{4 i}{3}\right)} \in X$.

On the other hand, $\lim _{n \rightarrow \infty} f z_{n}=\lim _{n \rightarrow \infty} g z_{n}=t=f u=u^{2}-\frac{i}{3} \Rightarrow u= \pm \sqrt{\left[t+\frac{i}{3}\right]}=$ $\pm \sqrt{\left[\left\{\frac{3}{2}-\frac{1}{2} \sqrt{\left(5+\frac{4 i}{3}\right)}\right\}+\frac{i}{3}\right]}$; both the value of $u$ gives for $\left(C L R_{f}\right)$.

Hence the pair $(f, g)$ also satisfy property $\left(C L R_{f}\right)$ in $X$ with

$u_{1}=\sqrt{\left[\left\{\frac{3}{2}-\frac{1}{2} \sqrt{\left(5+\frac{4 i}{3}\right)}\right\}+\frac{i}{3}\right]} \in X$

$u_{2}=\sqrt{\left[\left\{\frac{3}{2}-\frac{1}{2} \sqrt{\left(5+\frac{4 i}{3}\right)}\right\}+\frac{i}{3}\right]} \in X$.

For another sequence $w_{n}=\frac{-1}{2}-\frac{1}{2} \sqrt{\left(5+\frac{4 i}{3}\right)}$, the pair $(f, g)$ satisfy property $\left(C L R_{g}\right)$; and thus will satisfy $\left(C L R_{f}\right)$. Finally, we conclude that if the pair of self-mappings $f, g$ : $X \rightarrow X$ satisfy property $\left(C L R_{g}\right)$ then so is $\left(C L R_{f}\right)$, and vice-versa.

Now, we introduce the notion of (CLCS) property:

Definition 2.10. Suppose that $(X, d)$ be a complex valued b-metric space and $f, g: X \rightarrow X$. Let $Y \subseteq X$. The mappings $f, g$ are said to satisfy the property of "common limit converging in the range sub-space $Y$ ", in brief (CLCS) property in $Y$, if there exist a sequence $\left\{z_{n}\right\}$ in $X$ such that

$$
\lim _{n \rightarrow \infty} f z_{n}=\lim _{n \rightarrow \infty} g z_{n} \in Y
$$

for some sequence $\left\{z_{n}\right\}$ in $X$.

Remark 2.4. The $\left(C L R_{g}\right)$ and $\left(C L R_{f}\right)$ properties unify if $Y=f(X) \cap g(X)$.

Lemma 2.1. The continuity of one mapping with $(C L R)$ property of another mapping implies the (CLCS) property.

Proof. Let $f, g: X \rightarrow X$ and $f$ is continuous. Let $(f, g)$ is $\left(C L R_{g}\right)$ in $X$. Assume $\left\{z_{n}\right\}$ be a sequence in $X$. Then for $\left\{z_{n}\right\} \subseteq X$ we have $\lim _{n \rightarrow \infty} f z_{n}=f u=$ $\lim _{n \rightarrow \infty} g z_{n}=g v$, for some $u, v \in X$. Hence $(f, g)$ is $\left(C L R_{f}\right)$. So that $(f, g)$ is (CLCS) in $f(X) \cap g(X)$. Similar argument applies if $g$ is continuous and $(f, g)$ is $\left(C L R_{f}\right)$. This proves the Lemma with above Remark.

Following are some examples of (CLCS) property in metric spaces, complexvalued metric spaces and complex-valued $b$-metric spaces:

Example 2.8. Let $(X, d), X=\mathbb{C}$ be any complex valued metric space and $f, g$ : $X \rightarrow X$ be defined as $f z=\frac{z}{4}, g z=\frac{3 z}{4}, \forall z \in X$. Then for the sequence $\left\{z_{n}\right\}=\left\{\frac{1+i}{n}\right\}$, we have $\lim _{n \rightarrow \infty} f z_{n}=\lim _{n \rightarrow \infty} g z_{n}=0 \in Y=f(X) \cap g(X)$. Hence $(f, g)$ is (CLCS) in the subspace $Y=f(X) \cap g(X)=\mathbb{C}$. 
Example 2.9. Let $(X, d)$ be any complex valued metric space and $f, g: X \rightarrow X$ be defined as $f z=z+1, g z=2 z, \forall z \in X$. Then for the sequence $\left\{z_{n}\right\}=\left\{1+\frac{1}{n}\right\}$, we have $\lim _{n \rightarrow \infty} f z_{n}=\lim _{n \rightarrow \infty} g z_{n}=2 \in Y=f(X) \cap g(X)$. Hence $(f, g)$ is (CLCS) in the subspace $Y=f(X) \cap g(X)$.

Example 2.10. Let $(X, d)$ be a usual metric space and $f, g: X \rightarrow X=[0, \infty)$ be defined by: $f x=x^{2}+2, g x=2 x+10, \forall x \in X$. Then $f(X)=[2, \infty), g(X)=[10, \infty)$. Take a sequence $\left\{x_{n}\right\}=\left\{4-\frac{1}{n}\right\}$, then we have $\lim _{n \rightarrow \infty} f x_{n}=\lim _{n \rightarrow \infty} g x_{n}=18 \in$ $f(X) \cap g(X)=[10, \infty)=Y$. Hence $(f, g)$ is (CLCS) in $Y$.

Example 2.11. Let $(X, d)$ be a metric space with $d(x, y)=\frac{1}{4}|x-y|$. Define $f, g$ : $X \rightarrow X=[5,50]$ by: $f x=\frac{1}{2}(x+5), g x=\frac{1}{3}(x+15), \forall x \in X$. So that $f(X)=\left[5, \frac{55}{2}\right]$, $g(X)=\left[\frac{20}{3}, \frac{65}{3}\right)$. Then for the sequence $\left\{x_{n}\right\}=\left\{15+\frac{1}{n}\right\}$, we have $\lim _{n \rightarrow \infty} f x_{n}=$ $\lim _{n \rightarrow \infty} g x_{n}=10 \in f(X) \cap g(X)=\left[\frac{20}{3}, \frac{65}{3}\right)=Y$. Hence $(f, g)$ is (CLCS) in $Y$.

Example 2.12. Let $X=\mathbb{C}$. Define complex valued b-metric $d: X \times X \rightarrow \mathbb{C}$ by: $d(x, y)=|x-y|^{2}+i|x-y|^{2}, \forall x, y \in X$. Define mappings $f, g: X \rightarrow X$ by: $f x=\frac{x}{4}, g x=$ $\frac{x}{5}, \forall x \in X$. Then $f(X)=\mathbb{C}=g(X)=f(X) \cap g(X)=Y$ (say). Let $\left\{z_{n}\right\}=\left\{\frac{2+3 i}{5 n}\right\}$ be a sequence in $X$. Then $\lim _{n \rightarrow \infty} f z_{n}=\lim _{n \rightarrow \infty} g x_{n}=0 \in Y$. Hence $(f, g)$ is (CLCS) in $Y$.

Definition 2.11. [20] A pair of self-mappings $A, S: X \rightarrow X$ is called weakly compatible if they commute at their coincidence points. That is, if there be a point $u \in X$ such that $A u=S u$, then $A S u=S A u$, for each $u \in X$.

Example 2.13. Let $X=[0,1]$. Define complex valued b-metric $d: X \times X \rightarrow \mathbb{C}$ by: $d(x, y)=|x-y|^{2}+i|x-y|^{2}, \forall x, y \in X$. Define mappings $f, g: X \rightarrow X$ by: $f x=\frac{x}{4} ; g x=$ $\frac{x}{5}, \forall x \in X$. Then $f$ and $g$ have coincidence point at $x=0$. Now at this point, $f g 0=g f 0$. Thus $(f, g)$ is weakly compatible at 0 .

\section{Main Results}

Here is the main results:

\subsection{First main Theorem}

Theorem 3.1. Let $A, B, S, T: X \rightarrow X$ be four self-mappings of a complex valued b-metric space $(X, d)$ satisfying:

(i) The pair $(A, S)$ satisfy the (CLCS) property in $T(X)$, or $B(X)$; and another pair $(B, T)$ satisfy the (CLCS) property in $S(X)$ or $A(X)$,

(ii) points $x, y$ satisfy a set of rational inequalities

$$
d(A x, B y) \precsim q \cup_{x, y}(A, B, S, T), \forall x, y \in X
$$


where $q$ is a non-negative real number such that $0 \leq q<\frac{1}{s^{2}+s}$, with $s \geq 1$, and

$$
\begin{array}{r}
\cup_{x, y}(A, B, S, T) \in\left\{d(S x, T y), \frac{d(A x, S x) d(B y, T y)}{d(S x, T y)+d(A x, B y)},\right. \\
\frac{d(B y, S x) d(A x, T y)}{d(S x, T y)+d(A x, B y)}, \frac{d(A x, S x) d(B y, S x)}{1+d(S x, T y)}, \\
\frac{d(A x, T y) d(B y, T y)}{1+d(S x, T y)}, \frac{d(A x, S x) d(B y, T y)}{1+d(A x, B y)}, \frac{d(S x, B y) d(A x, B y)}{1+d(A x, B y)}, \\
\left.\frac{d(B y, S x) d(S x, T y)}{1+d(S x, T y)}, \frac{d(A x, S x) d(S x, T y)}{1+d(S x, T y)}, \frac{d(S x, T y) d(B y, T y)}{d(A x, B y)+d(S x, T y)}\right\},
\end{array}
$$

(iii) both the pairs $(A, S)$ and $(B, T)$ are weakly compatible.

Then mappings $A, B, S$ and $T$ have a unique common fixed point in $X$.

Proof. We take condition (i) one by one.

Case I. First suppose that the pair $(A, S)$ satisfy (CLCS) property in $T(X)$. Then, according to Definition 2.10, there exist a sequence $\left\{x_{n}\right\}$ in $X$ such that $\lim _{n \rightarrow \infty} A x_{n}=\lim _{n \rightarrow \infty} S x_{n}=t \in T X$. So, there exist $t \in T(X)$ such that $t=T v$ for some $v \in X$. We claim that $B v=t$, i.e., $d(B v, t)=0$. If not, then putting $x=x_{n}, y=v$ in (ii) we have

$$
d\left(A x_{n}, B v\right) \precsim q \cup_{x_{n}, v}(A, B, S, T),
$$

where $q$ is a non-negative real number such that $0 \leq q<\frac{1}{s^{2}+s}, s \geq 1$, and

$$
\begin{array}{r}
\cup_{x_{n}, v}(A, B, S, T) \in\left\{d\left(S x_{n}, T v\right), \frac{d\left(A x_{n}, S x_{n}\right) d(B v, T v)}{d\left(S x_{n}, T v\right)+d\left(A x_{n}, B v\right)},\right. \\
\frac{d\left(B v, S x_{n}\right) d\left(A x_{n}, T v\right)}{d\left(S x_{n}, T v\right)+d\left(A x_{n}, B v\right)}, \frac{d\left(A x_{n}, S x_{n}\right) d\left(B v, S x_{n}\right)}{1+d\left(S x_{n}, T v\right)}, \\
\frac{d\left(A x_{n}, T v\right) d(B v, T v)}{1+d\left(S x_{n}, T v\right)}, \frac{d\left(A x_{n}, S x_{n}\right) d(B v, T v)}{1+d\left(A x_{n}, B v\right)}, \\
\frac{d\left(S x_{n}, B v\right) d\left(A x_{n}, B v\right)}{1+d\left(A x_{n}, B v\right)}, \frac{d\left(B v, S x_{n}\right) d\left(S x_{n}, T v\right)}{1+d\left(S x_{n}, T v\right)}, \frac{d\left(A x_{n}, S x_{n}\right) d\left(S x_{n}, T v\right)}{1+d\left(S x_{n}, T v\right)}, \\
\left.\frac{d\left(S x_{n}, T v\right) d(B v, T v)}{d\left(A x_{n}, B v\right)+d\left(S x_{n}, T v\right)}\right\},
\end{array}
$$

We have following cases to consider:

sub-case 1. If $d\left(S x_{n}, T v\right)$ is chosen in (3.2), then we have

$$
d\left(A x_{n}, B v\right) \precsim q \cdot d\left(S x_{n}, T v\right) \Rightarrow\left|d\left(A x_{n}, B v\right)\right| \leq q \cdot\left|d\left(S x_{n}, T v\right)\right| .
$$

Letting $n \rightarrow \infty$ it yields: 


$$
\lim _{n \rightarrow \infty}|d(t, B v)|=\lim _{n \rightarrow \infty} q \cdot|d(t, t)|=0 \Rightarrow B v=t .
$$

sub-case 2. If $\frac{d\left(A x_{n}, S x_{n}\right) d(B v, T v)}{d\left(S x_{n}, T v\right)+d\left(A x_{n}, B v\right)}$ is chosen in (3.2), then we have

$$
\begin{aligned}
d\left(A x_{n}, B v\right) & \precsim q \cdot \frac{d\left(A x_{n}, S x_{n}\right) d(B v, T v)}{d\left(S x_{n}, T v\right)+d\left(A x_{n}, B v\right)} \\
\Rightarrow\left|d\left(A x_{n}, B v\right)\right| & \leq q \cdot \frac{\left|d\left(A x_{n}, S x_{n}\right)\right| \cdot \mid d(B v, T v)}{\left|d\left(S x_{n}, T v\right)+d\left(A x_{n}, B v\right)\right|}
\end{aligned}
$$

Letting $n \rightarrow \infty$ it yields:

$$
|d(t, B v)| \leq q \cdot \frac{|d(t, t)| \cdot \mid d(B v, t)}{|d(t, t)+d(t, B v)|}=0 \Rightarrow B v=t .
$$

sub-cases $3,4,5,6$. If

$\frac{d\left(B v, S x_{n}\right) d\left(A x_{n}, T v\right)}{d\left(S x_{n}, T v\right)+d\left(A x_{n}, B v\right)}$, or $\frac{d\left(A x_{n}, S x_{n}\right) d\left(B v, S x_{n}\right)}{1+d\left(S x_{n}, T v\right)}$, or $\frac{d\left(A x_{n}, T v\right) d(B v, T v)}{1+d\left(S x_{n}, T v\right)}$, or $\frac{d\left(A x_{n}, S x_{n}\right) d(B v, T v)}{1+d\left(A x_{n}, B v\right)}$, is chosen in (3.2), then as in subcases 2 , on letting $n \rightarrow \infty$, we have

$$
\lim _{n \rightarrow \infty}\left|d\left(A x_{n}, B v\right)\right|=q .0=0 \Rightarrow B v=t
$$

sub-case 7. If $\frac{d\left(S x_{n}, B v\right) d\left(A x_{n}, B v\right)}{1+d\left(A x_{n}, B v\right)}$ is chosen in (3.2), then we have

$$
\begin{array}{r}
d\left(A x_{n}, B v\right) \precsim q \cdot \frac{d\left(S x_{n}, B v\right) d\left(A x_{n}, B v\right)}{1+d\left(A x_{n}, B v\right)} \\
\Rightarrow\left|d\left(A x_{n}, B v\right)\right| \leq q \cdot \frac{\left|d\left(S x_{n}, B v\right)\right| \cdot\left|d\left(A x_{n}, B v\right)\right|}{\left|1+d\left(A x_{n}, B v\right)\right|} .
\end{array}
$$

Letting $n \rightarrow \infty$, and using $|1+d(t, B v)|<|d(t, B v)|$ it yields:

$$
\begin{array}{r}
|d(t, B v)| \leq q \cdot \frac{|d(t, B v)| \cdot|d(t, B v)|}{|1+d(t, B v)|} \\
<q \cdot \frac{|d(t, B v)| \cdot|d(t, B v)|}{|d(t, B v)|}=q \cdot|d(t, B v)|<|d(t, B v)|,
\end{array}
$$

a contradiction. Thus $B v=t$. 
sub-case 8. If $\frac{d\left(B v, S x_{n}\right) d\left(S x_{n}, T v\right)}{1+d\left(S x_{n}, T v\right)}$ is chosen in (3.2), then we have

$$
\begin{array}{r}
d\left(A x_{n}, B v\right) \precsim q \cdot \frac{d\left(B v, S x_{n}\right) d\left(S x_{n}, T v\right)}{1+d\left(S x_{n}, T v\right)} \\
\Rightarrow\left|d\left(A x_{n}, B v\right)\right| \leq q \frac{\left|d\left(B v, S x_{n}\right)\right| \cdot\left|d\left(S x_{n}, T v\right)\right|}{\left|1+d\left(S x_{n}, T v\right)\right|} .
\end{array}
$$

Letting $n \rightarrow \infty$ it yields

$$
\lim _{n \rightarrow \infty}\left|d\left(A x_{n}, B v\right)\right|=|d(t, B v)| \leq q \cdot \lim _{n \rightarrow \infty} \frac{|d(B v, t)| \cdot|d(t, t)|}{|1+d(t, t)|}=q .0 \Rightarrow B v=t
$$

sub-case 9. If $\frac{d\left(A x_{n}, S x_{n}\right) d\left(S x_{n}, T v\right)}{1+d\left(S x_{n}, T v\right)}$ is chosen in (3.2), then we have

$$
\begin{array}{r}
d\left(A x_{n}, B v\right) \precsim q \cdot \frac{d\left(A x_{n}, S x_{n}\right) d\left(S x_{n}, T v\right)}{1+d\left(S x_{n}, T v\right)} \\
\Rightarrow\left|d\left(A x_{n}, B v\right)\right| \leq q \cdot \frac{\left|d\left(A x_{n}, S x_{n}\right)\right| \cdot\left|d\left(S x_{n}, T v\right)\right|}{\left|1+d\left(S x_{n}, T v\right)\right|} .
\end{array}
$$

Letting $n \rightarrow \infty$, and since $\lim _{n \rightarrow \infty} A x_{n}=\lim _{n \rightarrow \infty} S x_{n}=t=T v$ it yields:

$$
\begin{array}{r}
\lim _{n \rightarrow \infty}\left|d\left(A x_{n}, B v\right)\right|=|d(t, B v)| \leq q . \lim _{n \rightarrow \infty} \frac{\left|d\left(A x_{n}, S x_{n}\right)\right| \cdot\left|d\left(S x_{n}, T v\right)\right|}{\left|1+d\left(S x_{n}, T v\right)\right|} \\
=q \cdot \frac{|d(t, t)| \cdot|d(t, t)|}{|1+d(t, t)|}=q .0
\end{array}
$$

Thus $B v=t$.

sub-case 10. If $\frac{d\left(S x_{n}, T v\right) d(B v, T v)}{d\left(A x_{n}, B v\right)+d\left(S x_{n}, T v\right)}$ is chosen in (3.2), then we have

$$
\begin{array}{r}
d\left(A x_{n}, B v\right) \precsim q \cdot \frac{d\left(S x_{n}, T v\right) d(B v, T v)}{d\left(A x_{n}, B v\right)+d\left(S x_{n}, T v\right)} \\
\Rightarrow\left|d\left(A x_{n}, B v\right)\right| \leq q \cdot \frac{\left|d\left(S x_{n}, T v\right)\right| \cdot|d(B v, T v)|}{\left|d\left(A x_{n}, B v\right)+d\left(S x_{n}, T v\right)\right|} .
\end{array}
$$

Letting $n \rightarrow \infty$ it yields:

$$
\begin{array}{r}
\lim _{n \rightarrow \infty}\left|d\left(A x_{n}, B v\right)\right|=|d(t, B v)|=q \cdot \lim _{n \rightarrow \infty} \frac{\left|d\left(S x_{n}, T v\right)\right| \cdot|d(B v, T v)|}{\left|d\left(A x_{n}, B v\right)+d\left(S x_{n}, T v\right)\right|} \\
=q \cdot \frac{|d(t, t)| \cdot|d(B v, t)|}{|d(t, B v)+d(t, t)|}=q .0 \Rightarrow B v=t .
\end{array}
$$


Therefore, in all cases, we obtain $B v=t$. Hence

$$
\lim _{n \rightarrow \infty} A x_{n}=\lim _{n \rightarrow \infty} S x_{n}=B v=T v=t .
$$

Hence $v$ is a coincidence point of $(B, T)$. Now, the weakly compatibility of pair $(B, T)$ implies that $T B v=B T v=T t=B t$. Next, we claim that $t$ is a common fixed point of $(B, T)$, i.e., $B t=T t=t$. For, putting $x=x_{n}, y=t$ in condition (ii) and using $\lim _{n \rightarrow \infty} A x_{n}=t=\lim _{n \rightarrow \infty} S x_{n}, T t=B t$, we have

$$
d\left(A x_{n}, B t\right) \precsim q \cup_{x_{n}, t}(A, B, S, T),
$$

where $q$ is a non-negative real number such that $0 \leq q<\frac{1}{s^{2}+s}, s \geq 1$, and

$$
\begin{array}{r}
\cup_{x_{n}, t}(A, B, S, T) \in\left\{d\left(S x_{n}, T t\right), \frac{d\left(A x_{n}, S x_{n}\right) d(B t, T t)}{d\left(S x_{n}, T t\right)+d\left(A x_{n}, B t\right)},\right. \\
\frac{d\left(B t, S x_{n}\right) d\left(A x_{n}, T t\right)}{d\left(S x_{n}, T t\right)+d\left(A x_{n}, B t\right)}, \frac{d\left(A x_{n}, S x_{n}\right) d\left(B t, S x_{n}\right)}{1+d\left(S x_{n}, T t\right)}, \frac{d\left(A x_{n}, T t\right) d(B t, T t)}{1+d\left(S x_{n}, T t\right)}, \\
\frac{d\left(A x_{n}, S x_{n}\right) d(B t, T t)}{1+d\left(A x_{n}, B t\right)}, \frac{d\left(S x_{n}, B t\right) d\left(A x_{n}, B t\right)}{1+d\left(A x_{n}, B t\right)}, \\
\left.\frac{d\left(B t, S x_{n}\right) d\left(S x_{n}, T t\right)}{1+d\left(S x_{n}, T t\right)}, \frac{d\left(A x_{n}, S x_{n}\right) d\left(S x_{n}, T t\right)}{1+d\left(S x_{n}, T t\right)}, \frac{d\left(S x_{n}, T t\right) d(B t, T t)}{d\left(A x_{n}, B t\right)+d\left(S x_{n}, T t\right)}\right\},
\end{array}
$$

We have the following cases to consider:

sub-case 1. If $d\left(S x_{n}, T t\right)$ is chosen in (3.4), then we have

$$
d\left(A x_{n}, B t\right) \precsim q \cdot d\left(S x_{n}, T t\right) \Rightarrow\left|d\left(A x_{n}, B t\right)\right| \leq q \cdot\left|d\left(S x_{n}, T t\right)\right|=q \cdot\left|d\left(S x_{n}, B t\right)\right| .
$$

Letting $n \rightarrow \infty$, it yields

$$
\lim _{n \rightarrow \infty}\left|d\left(A x_{n}, B t\right)\right|=|d(t, B t)| \leq q . \lim _{n \rightarrow \infty}\left|d\left(S x_{n}, B t\right)\right|<|d(t, B t)|,
$$

a contradiction. Thus $B t=t$.

sub-case $2,5,6,10$. If

$$
\begin{aligned}
\frac{d\left(A x_{n}, S x_{n}\right) d(B t, T t)}{d\left(S x_{n}, T t\right)+d\left(A x_{n}, B t\right)} & =0=\frac{d\left(A x_{n}, T t\right) d(B t, T t)}{1+d\left(S x_{n}, T t\right)}=\frac{d\left(A x_{n}, S x_{n}\right) d(B t, T t)}{1+d\left(A x_{n}, B t\right)} \\
& =\frac{d\left(S x_{n}, T t\right) d(B t, T t)}{d\left(A x_{n}, B t\right)+d\left(S x_{n}, T t\right)}
\end{aligned}
$$


is chosen in (3.4), then it follows that

$$
d\left(A x_{n}, B t\right) \precsim q .0 \Rightarrow\left|d\left(A x_{n}, B t\right)\right| \leq 0 .
$$

Letting $n \rightarrow \infty$, it yields

$$
\lim _{n \rightarrow \infty}\left|d\left(A x_{n}, B t\right)\right|=|d(t, B t)| \leq 0
$$

Thus $B t=t$.

sub-case 3. If $\frac{d\left(B t, S x_{n}\right) d\left(A x_{n}, T t\right)}{d\left(S x_{n}, T t\right)+d\left(A x_{n}, B t\right)}$ is chosen in (3.4), then we have

$$
\begin{array}{r}
d\left(A x_{n}, B t\right) \precsim q \cdot \frac{d\left(B t, S x_{n}\right) d\left(A x_{n}, T t\right)}{d\left(S x_{n}, T t\right)+d\left(A x_{n}, B t\right)} \Rightarrow\left|d\left(A x_{n}, B t\right)\right| \\
\leq q \cdot \frac{\left|d\left(B t, S x_{n}\right)\right| \cdot\left|d\left(A x_{n}, T t\right)\right|}{\left|d\left(S x_{n}, T t\right)+d\left(A x_{n}, B t\right)\right|} .
\end{array}
$$

Letting $n \rightarrow \infty$, it yields

$$
\begin{aligned}
\lim _{n \rightarrow \infty}\left|d\left(A x_{n}, B t\right)\right| & =|d(t, B t)| \leq q \cdot \lim _{n \rightarrow \infty} \frac{\left|d\left(B t, S x_{n}\right)\right| \cdot\left|d\left(A x_{n}, T t\right)\right|}{\left|d\left(S x_{n}, T t\right)+d\left(A x_{n}, B t\right)\right|} \\
& =\frac{q}{2}|d(t, B t)|,
\end{aligned}
$$

a contradiction. Thus $B t=t$.

sub-case 4. If $\frac{d\left(A x_{n}, S x_{n}\right) d\left(B t, S x_{n}\right)}{1+d\left(S x_{n}, T t\right)}$ is chosen in (3.4), then we have

$$
\begin{array}{r}
d\left(A x_{n}, B t\right) \\
\precsim q \cdot \frac{d\left(A x_{n}, S x_{n}\right) d\left(B t, S x_{n}\right)}{1+d\left(S x_{n}, T t\right)} \\
\Rightarrow\left|d\left(A x_{n}, B t\right)\right| \leq q \cdot \frac{\left|d\left(A x_{n}, S x_{n}\right)\right| \cdot\left|d\left(B t, S x_{n}\right)\right|}{\left|1+d\left(S x_{n}, T t\right)\right|} .
\end{array}
$$

Letting $n \rightarrow \infty$, it yields

$$
\begin{aligned}
\lim _{n \rightarrow \infty}\left|d\left(A x_{n}, B t\right)\right| & =|d(t, B t)| \leq q \cdot \lim _{n \rightarrow \infty} \frac{\left|d\left(A x_{n}, S x_{n}\right)\right| \cdot\left|d\left(B t, S x_{n}\right)\right|}{\left|1+d\left(S x_{n}, T t\right)\right|} \\
& =q \cdot \frac{|d(t, t)| \cdot|d(B t, t)|}{|1+d(t, T t)|}=q \cdot 0=0 .
\end{aligned}
$$

Thus $B t=t$. 
sub-case 7. If $\frac{d\left(S x_{n}, B t\right) d\left(A x_{n}, B t\right)}{1+d\left(A x_{n}, B t\right)}$ is chosen in (3.4), then we have

$$
\begin{aligned}
& d\left(A x_{n}, B t\right) \precsim q \cdot \frac{d\left(S x_{n}, B t\right) d\left(A x_{n}, B t\right)}{1+d\left(A x_{n}, B t\right)} \\
& \Rightarrow\left|d\left(A x_{n}, B t\right)\right| \leq q \cdot \frac{\left|d\left(S x_{n}, B t\right)\right| \cdot\left|d\left(A x_{n}, B t\right)\right|}{\left|1+d\left(A x_{n}, B t\right)\right|} .
\end{aligned}
$$

Letting $n \rightarrow \infty$, it yields

$$
\begin{array}{r}
\lim _{n \rightarrow \infty}\left|d\left(A x_{n}, B t\right)\right|=|d(t, B t)| \leq \\
q . \lim _{n \rightarrow \infty} \frac{\left|d\left(S x_{n}, B t\right)\right| \cdot\left|d\left(A x_{n}, B t\right)\right|}{\left|1+d\left(A x_{n}, B t\right)\right|}=\frac{|d(t, B t)| \cdot|d(t, B t)|}{|1+d(t, B t)|} .
\end{array}
$$

But $|1+d(t, B t)|>|d(t, B t)|$, so that

$$
|d(t, B t)| \leq q \cdot \frac{|d(t, B t)| \cdot|d(t, B t)|}{|1+d(t, B t)|}<|d(t, B t)|,
$$

a contradiction. Thus $B t=t$.

sub-case 8. If $\frac{d\left(B t, S x_{n}\right) d\left(S x_{n}, T t\right)}{1+d\left(S x_{n}, T t\right)}$ is chosen in (3.4), then we have

$$
\begin{aligned}
& d\left(A x_{n}, B t\right) \precsim q \cdot \frac{d\left(B t, S x_{n}\right) d\left(S x_{n}, T t\right)}{1+d\left(S x_{n}, T t\right)} \\
& \Rightarrow\left|d\left(A x_{n}, B t\right)\right| \leq q \cdot \frac{\left|d\left(B t, S x_{n}\right)\right| \cdot\left|d\left(S x_{n}, T t\right)\right|}{\left|1+d\left(S x_{n}, T t\right)\right|} .
\end{aligned}
$$

Letting $n \rightarrow \infty$, it yields

$$
\begin{aligned}
\lim _{n \rightarrow \infty}\left|d\left(A x_{n}, B t\right)\right|=|d(t, B t)| \leq q \cdot \lim _{n \rightarrow \infty} \frac{\left|d\left(B t, S x_{n}\right)\right| \cdot\left|d\left(S x_{n}, T t\right)\right|}{\left|1+d\left(S x_{n}, T t\right)\right|} \\
=q \cdot \frac{|d(B t, t)| \cdot|d(t, T t)|}{|1+d(t, T t)|} .
\end{aligned}
$$

But $|1+d(t, T t)|>|d(t, T t)|$; so above yields

$$
|d(t, B t)| \leq q \cdot \frac{|d(B t, t)| \cdot|d(t, T t)|}{|1+d(t, T t)|}<|d(B t, t)|,
$$

a contradiction. Thus $B t=t$. 
sub-case 9. If $\frac{d\left(A x_{n}, S x_{n}\right) d\left(S x_{n}, T t\right)}{1+d\left(S x_{n}, T t\right)}$ is chosen in (3.4), then we have

$$
\begin{array}{r}
d\left(A x_{n}, B t\right) \\
\precsim q \cdot \frac{d\left(A x_{n}, S x_{n}\right) d\left(S x_{n}, T t\right)}{1+d\left(S x_{n}, T t\right)} \\
\Rightarrow\left|d\left(A x_{n}, B t\right)\right| \leq q \cdot \frac{\left|d\left(A x_{n}, S x_{n}\right)\right| \cdot\left|d\left(S x_{n}, T t\right)\right|}{\left|1+d\left(S x_{n}, T t\right)\right|} .
\end{array}
$$

Letting $n \rightarrow \infty$, it yields

$$
\lim _{n \rightarrow \infty}\left|d\left(A x_{n}, B t\right)\right|=|d(t, B t)| \leq q . \lim _{n \rightarrow \infty} \frac{\left|d\left(A x_{n}, S x_{n}\right)\right| .\left|d\left(S x_{n}, T t\right)\right|}{\left|1+d\left(S x_{n}, T t\right)\right|}=q .0=0 .
$$

Thus $B t=t$. Hence in all cases, $B t=T t=t$. It shows that $t \in T(X)$ is a common fixed point of $(B, T)$.

Case II. A similar argument arises if the same pair $(A, S)$ as in Case I, satisfies (CLCS) property in another range subspace $B(X)$. In this case $t \in B(X)$ is a common fixed point of $(B, T)$.

Case III. Next, suppose that the second pair $(B, T)$ satisfies (CLCS) property in a subspace $S(X)$. Then, according to Definition 2.10, there exists a sequence $\left\{y_{n}\right\}$ in $X$ such that $\lim _{n \rightarrow \infty} B y_{n}=\lim _{n \rightarrow \infty} T y_{n} \in S(X)$. So, there exist $t^{\prime} \in S(X)$ such that $t^{\prime}=S u$ for some $u \in X$, where $t^{\prime}=\lim _{n \rightarrow \infty} B y_{n}=\lim _{n \rightarrow \infty} T y_{n}$. The claim $A u=t^{\prime}$ follows exactly as in Case I. It shows that $u$ is a coincidence point of $(A, S)$. The weakly compatibility of $(A, S)$ implies that $A S u=S A u=A t^{\prime}=S t^{\prime}$. It shows that $t^{\prime}$ is a coincidence point of $(A, S)$ and $t \in S(X)$.

Now, we claim that $t^{\prime}$ is a common fixed point of $(A, S)$. This follows exactly as in Case I, by putting $x=t^{\prime}, y=y_{n}$ in condition (ii), making $n \rightarrow \infty$, and using $A t^{\prime}=S t^{\prime}$. Hence $A t^{\prime}=t^{\prime}$. It shows that $t^{\prime} \in S(X)$ is a common fixed point of $(A, S)$.

Case IV. A similar argument arises if the second pair $(B, T)$ satisfy the (CLCS) property in range subspace $A(X)$. In this case $t^{\prime} \in A(X)$ is a common fixed point of $(A, S)$.

Further, we claim that the common fixed point $t^{\prime}$ of $(A, S)$, and $t$ of $(B, T)$ are same, i.e., $t=t^{\prime}$. If not, then putting $x=t^{\prime}, y=t$ in condition (ii), and using $A t^{\prime}=S t^{\prime}=t^{\prime}, B t=T t=t$, we have

$$
d\left(A t^{\prime}, B t\right)=d\left(t^{\prime}, t\right) \precsim q \cup_{t^{\prime}, t}(A, B, S, T)
$$

where $q$ is a non-negative real number such that $0 \leq q<\frac{1}{s^{2}+s}, s \geq 1$, and 


$$
\begin{array}{r}
\cup_{t^{\prime}, t}(A, B, S, T) \in\left\{d\left(S t^{\prime}, T t\right), \frac{d\left(A t^{\prime}, S t^{\prime}\right) d(B t, T t)}{d\left(S t^{\prime}, T t\right)+d\left(A t^{\prime}, B t\right)}, \frac{d\left(B t, S t^{\prime}\right) d\left(A t^{\prime}, T t\right)}{d\left(S t^{\prime}, T t\right)+d\left(A t^{\prime}, B t\right)},\right. \\
\frac{d\left(A t^{\prime}, S t^{\prime}\right) d\left(B t, S t^{\prime}\right)}{1+d\left(S t^{\prime}, T t\right)}, \frac{d\left(A t^{\prime}, T t\right) d(B t, T t)}{1+d\left(S t^{\prime}, T t\right)}, \\
\frac{d\left(A t^{\prime}, S t^{\prime}\right) d(B t, T t)}{1+d\left(A t^{\prime}, B t^{\prime}\right)}, \frac{d\left(S t^{\prime}, B t\right) d\left(A t^{\prime}, B t\right)}{1+d\left(A t^{\prime}, B t\right)}, \\
\left.\frac{d\left(B t, S t^{\prime}\right) d\left(S t^{\prime}, T t\right)}{1+d\left(S t^{\prime}, T t\right)}, \frac{d\left(A t^{\prime}, S t^{\prime}\right) d\left(S t^{\prime}, T t\right)}{1+d\left(S t^{\prime}, T t\right)}, \frac{d\left(S t^{\prime}, T t\right) d(B t, T t)}{d\left(A t^{\prime}, B t\right)+d\left(S t^{\prime}, T t\right)}\right\}, \\
=\left\{d\left(t^{\prime}, t\right), 0, \frac{1}{2} d\left(t, t^{\prime}\right), 0,0,0, \frac{d\left(t^{\prime}, t\right) d\left(t^{\prime}, t\right)}{1+d\left(t^{\prime}, t\right)}, \frac{d\left(t, t^{\prime}\right) d\left(t^{\prime}, t\right)}{1+d\left(t^{\prime}, t\right)}, 0,0\right\} .
\end{array}
$$

Here arises following cases:

sub-case-1. If $d\left(t^{\prime}, t\right)$ is chosen, then from (3.5), we have

$$
d\left(t^{\prime}, t\right) \precsim q \cdot d\left(t^{\prime}, t\right) \Rightarrow\left|d\left(t^{\prime}, t\right)\right|<q\left|d\left(t^{\prime}, t\right)\right|<\left|d\left(t^{\prime}, t\right)\right|,
$$

a contradiction. Thus $t=t^{\prime}$.

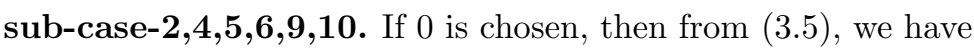

$$
d\left(t^{\prime}, t\right) \precsim q .0 \Rightarrow\left|d\left(t^{\prime}, t\right)\right| \leq 0 \text {. Thus } t=t^{\prime} .
$$

sub-case-3. If $\frac{1}{2} d\left(t^{\prime}, t\right)$ is chosen, then from (3.5), we have

$$
d\left(t^{\prime}, t\right) \precsim \frac{q}{2} \cdot d\left(t^{\prime}, t\right) \Rightarrow\left|d\left(t^{\prime}, t\right)\right| \leq \frac{q}{2}\left|d\left(t^{\prime}, t\right)\right|<\left|d\left(t^{\prime}, t\right)\right|,
$$

a contradiction. Thus $t=t^{\prime}$.

sub-case-7,8. If $\frac{d\left(t^{\prime}, t\right) d\left(t^{\prime}, t\right)}{1+d\left(t^{\prime}, t\right)}$ is chosen, then from (3.5), we have

$$
\begin{array}{r}
d\left(t^{\prime}, t\right) \precsim q \cdot \frac{d\left(t^{\prime}, t\right) d\left(t^{\prime}, t\right)}{1+d\left(t^{\prime}, t\right)} \\
\Rightarrow\left|d\left(t^{\prime}, t\right)\right| \leq q \cdot \frac{\left|d\left(t^{\prime}, t\right)\right| \cdot\left|d\left(t^{\prime}, t\right)\right|}{\left|1+d\left(t^{\prime}, t\right)\right|}<q \cdot\left|d\left(t^{\prime}, t\right)\right|,
\end{array}
$$

a contradiction, as $\left|1+d\left(t^{\prime}, t\right)\right|>\left|d\left(t^{\prime}, t\right)\right|$. Thus $t=t^{\prime}$.

Hence in all cases $t=t^{\prime}$. This shows that $t$ is a common fixed point of $A, B, S, T$. Uniqueness of common fixed point follows easily. This completes the proof. 
Remark 3.1. The condition (i) can be written as:

"the pair $(A, S)$ satisfy $\left(C L R_{T}\right)$, or $\left(C L R_{T}\right)$; and the pair $(B, T)$ satisfy $\left(C L R_{A}\right)$, or $\left(C L R_{S}\right)$ ".

So, taking this condition with condition (ii) and (iii), we get the fixed point result in terms of (CLR) property.

Remark 3.2. From case-I, II, III and IV, we observe that the unique common fixed point belongs to that range-subset in which the common converging point lies.

\subsection{Second main Theorem}

If one pair of mappings satisfy the (CLCS) property in the common range-subset of the second pair, and the second pair of mappings satisfy the (CLCS) property in the common range-subset of the first pair, then we have the following result:

Theorem 3.2. Let $A, B, S, T: X \rightarrow X$ be four self-mappings of a complex valued $b$-metric space $(X, d)$ satisfying:

(i) The first pair $(A, S)$ satisfy $(C L C S)$ property in $T(X) \cap B(X)$; and the second pair $(B, T)$ satisfy (CLCS) property in $S(X) \bigcap A(X)$,

(ii) $x, y$ satisfy a set of rational inequalities

$$
d(A x, B y) \precsim q \cup_{x, y}(A, B, S, T), \forall x, y \in X
$$

where $q$ is a non-negative real number such that $0 \leq q<\frac{1}{s^{2}+s}$, with $s \geq 1$, and

$$
\begin{array}{r}
\cup_{x, y}(A, B, S, T) \in\left\{d(S x, T y), \frac{d(A x, S x) d(B y, T y)}{d(S x, T y)+d(A x, B y)}, \frac{d(B y, S x) d(A x, T y)}{d(S x, T y)+d(A x, B y)},\right. \\
\frac{d(A x, S x) d(B y, S x)}{1+d(S x, T y)}, \frac{d(A x, T y) d(B y, T y)}{1+d(S x, T y)}, \\
\frac{d(A x, S x) d(B y, T y)}{1+d(A x, B y)}, \frac{d(S x, B y) d(A x, B y)}{1+d(A x, B y)}, \\
\left.\frac{d(B y, S x) d(S x, T y)}{1+d(S x, T y)}, \frac{d(A x, S x) d(S x, T y)}{1+d(S x, T y)}, \frac{d(S x, T y) d(B y, T y)}{d(A x, B y)+d(S x, T y)}\right\},
\end{array}
$$

(iii) both the pairs $(A, S)$ and $(B, T)$ are weakly compatible,

Then mappings $A, B, S$ and $T$ have a unique common fixed point in $X$.

Proof. The proof runs exactly as Theorem 3.1. Case I and Case II are merged, so the point $t^{\prime} \in B(X) \cap T(X)$. Similarly, Case III and Case IV are merged, so the point $t \in A(X) \cap S(X)$. Uniqueness follows easily. This completes the proof.

\subsection{Third Theorem}

If the (E.A) property is taken into consideration and the range subspaces are closed then we have the following result: 
Theorem 3.3. Let $A, B, S, T: X \rightarrow X$ be four self-mappings of a complex valued $b$-metric space $(X, d)$ satisfying:

(i) The pair $(A, S)$ satisfy (E.A) property and $T(X)$ is closed, or $B(X)$ is closed; and

the pair $(B, T)$ satisfy the (E.A) property and $S(X)$ is closed, or $A(X)$ is closed, (ii) $x, y$ satisfy a set of rational inequalities

$$
d(A x, B y) \precsim q \cup_{x, y}(A, B, S, T), \forall x, y \in X
$$

where $q$ is a non-negative real number such that $0 \leq q<\frac{1}{s^{2}+s}$, with $s \geq 1$, and

$$
\begin{array}{r}
\cup_{x, y}(A, B, S, T) \in\left\{d(S x, T y), \frac{d(A x, S x) d(B y, T y)}{d(S x, T y)+d(A x, B y)}, \frac{d(B y, S x) d(A x, T y)}{d(S x, T y)+d(A x, B y)},\right. \\
\frac{d(A x, S x) d(B y, S x)}{1+d(S x, T y)}, \frac{d(A x, T y) d(B y, T y)}{1+d(S x, T y)}, \\
\frac{d(A x, S x) d(B y, T y)}{1+d(A x, B y)}, \frac{d(S x, B y) d(A x, B y)}{1+d(A x, B y)}, \\
\left.\frac{d(B y, S x) d(S x, T y)}{1+d(S x, T y)}, \frac{d(A x, S x) d(S x, T y)}{1+d(S x, T y)}, \frac{d(S x, T y) d(B y, T y)}{d(A x, B y)+d(S x, T y)}\right\},
\end{array}
$$

(iii) both the pairs $(A, S)$ and $(B, T)$ are weakly compatible.

Then mappings $A, B, S$ and $T$ have a unique common fixed point in $X$.

Proof. The theorem exactly runs as Theorem 3.1. The point of common convergence belongs to the range subspaces, as in Case-I, II, III and IV. Rest part is same. This completes the proof.

If $A=B=f$ and $S=T=g$ in Theorem 3.1, we have the following result:

Corollary 3.1. Let $f, g: X \rightarrow X$ be two self-mappings of a complex valued $b$ metric space $(X, d)$ such that:

(i) The pair $(f, g)$ satisfy the (CLCS) property in $g(X)$, or $f(X)$

(ii)

$$
d(f x, f y) \precsim q \cup_{x, y}(f, g), \forall x, y \in X
$$

where $q$ is a non-negative real number such that $0 \leq q<\frac{1}{s^{2}+s}, s \geq 1$, and 


$$
\begin{array}{r}
\cup_{x, y}(f, g) \in\left\{d(g x, g y), \frac{d(f x, g x) d(f y, g y)}{d(g x, g y)+d(f x, f y)}, \frac{d(f y, g x) d(f x, g y)}{d(g x, g y)+d(f x, f y)},\right. \\
\frac{d(f x, g x) d(f y, g x)}{1+d(g x, g y)}, \frac{d(f x, g y) d(f y, g y)}{1+d(g x, g y)}, \\
\frac{d(f x, g x) d(f y, g y)}{1+d(f x, f y)}, \frac{d(g x, f y) d(f x, f y)}{1+d(f x, f y)}, \frac{d(f y, g x) d(g x, g y)}{1+d(g x, g y)}, \\
\left.\frac{d(f x, g x) d(g x, g y)}{1+d(g x, g y)}, \frac{d(g x, g y) d(f y, g y)}{d(f x, f y)+d(g x, g y)}\right\} .
\end{array}
$$

If the pair $(f, g)$ is weakly compatible and satisfy property (E.A), then mappings $f, g$ have a unique common fixed point in $X$.

Remark 3.3. The Corollary 3.1 is true for both condition, $\left(C L R_{g}\right)$ and $\left(C L R_{f}\right)$.

\subsection{Examples}

Following example validates our first main theorem:

Example 3.1. Let $X=\mathbb{C}$ is the set of complex numbers. Define $b$-metric $d: X \times X \rightarrow \mathbb{C}$ with the parameter $s=2$, by $d\left(z_{1}, z_{2}\right)=(1+\iota)\left|z_{1}-z_{2}\right|^{2}$, for all $z_{1}, z_{2} \in \mathbb{C}$. Obviously, $(X, d)$ is a complex-valued $b$-metric space. Define self-mappings $A, B, S, T: X \rightarrow X$ by:

$$
A z=\frac{z}{6}, \quad B z=\frac{z}{5}, \quad S z=\frac{-z}{2}, \quad T z=\frac{z}{2}, \quad \forall z \in \mathbb{C} .
$$

Observe that $A(X)=B(X)=S(X)=T(X)=\mathbb{C}$. Take a sequence $\left\{z_{n}\right\}=\left\{\frac{2+3 \iota}{5 n}\right\}$ in $X$. Then $\lim _{n \rightarrow \infty} A z_{n}=\lim _{n \rightarrow \infty} S z_{n}=0 \in T(X)=\mathbb{C}$, and also the limit $0 \in B(X)=\mathbb{C}$. Hence the pair $(A, S)$ satisfy the (CLCS) property in $T(X), B(X)$. Also, the pair $(A, S)$ and $(B, T)$ are weakly compatible at $z=0 \in \mathbb{C}$. Now, we discuss the inequality in following cases:

Case-1. $x=0, y \neq 0$ then

$d(S x, B y)=(1+\iota)|y|^{2} / 25, \quad d(A x, T y)=(1+\iota)|y|^{2} / 4, \quad d(A x, S x)=0, \quad d(B y, T y)=$ $9(1+\iota)|y|^{2} / 100, \quad d(A x, B y)=(1+\iota)|y|^{2} / 25$ and $d(S x, T y)=(1+\iota)|y|^{2} / 4$. Thus, the given inequality (3.1): $d(A x, B y) \precsim q \cup_{x, y}(A, B, S, T)$, where

$$
\begin{array}{r}
\cup_{x, y}(A, B, S, T) \in\left\{d(S x, T y), \frac{d(A x, S x) d(B y, T y)}{d(S x, T y)+d(A x, B y)},\right. \\
\frac{d(B y, S x) d(A x, T y)}{d(S x, T y)+d(A x, B y)}, \frac{d(A x, S x) d(B y, S x)}{1+d(S x, T y)}, \\
\frac{d(A x, T y) d(B y, T y)}{1+d(S x, T y)}, \frac{d(A x, S x) d(B y, T y)}{1+d(A x, B y)}, \frac{d(S x, B y) d(A x, B y)}{1+d(A x, B y)}, \\
\left.\frac{d(B y, S x) d(S x, T y)}{1+d(S x, T y)}, \frac{d(A x, S x) d(S x, T y)}{1+d(S x, T y)}, \frac{d(S x, T y) d(B y, T y)}{d(A x, B y)+d(S x, T y)}\right\} .
\end{array}
$$

reduces to: 


$$
\begin{array}{r}
\frac{(1+\iota)|y|^{2}}{25} \precsim q\left\{\frac{(1+\iota)|y|^{2}}{4}, 0, \frac{(1+\iota)|y|^{2}}{41}, 0, \frac{9(1+\iota)^{2}|y|^{4}}{400\left[1+(1+\iota)|y|^{2} / 4\right]}, 0,\right. \\
\left.\frac{25(1+\iota)^{2}|y|^{4}}{\left[1+(1+\iota)|y|^{2}\right]}, \frac{(1+\iota)^{2}|y|^{4}}{25\left[4+(1+\iota)|y|^{2}\right]}, 0, \frac{9(1+\iota)|y|^{2}}{116}\right\},
\end{array}
$$

This may be valid for at least one $q$, for which $0 \leq q<\frac{1}{s^{2}+s}=\frac{1}{6}$. For instance, $\frac{1}{6}>q \geq \frac{4}{25}$.

Case-2. $y=0, x \neq 0$. In this case, $B y=T y=0$ and $A x=\frac{x}{6}, S x=\frac{-x}{2}$, so that $d(A x, B y)=\frac{(1+\iota)|x|^{2}}{36}=d(A x, T y), d(S x, T y)=\frac{(1+\iota)|x|^{2}}{4}=d(B y, S x)$, $d(A x, S x)=\frac{4(1+\iota)|x|^{2}}{9}, d(B y, T y)=0$.

Thus, the given inequality (3.1): $d(A x, B y) \precsim q \cup_{x, y}(A, B, S, T)$, where

$$
\begin{array}{r}
\cup_{x, y}(A, B, S, T) \in\left\{d(S x, T y), \frac{d(A x, S x) d(B y, T y)}{d(S x, T y)+d(A x, B y)}, \frac{d(B y, S x) d(A x, T y)}{d(S x, T y)+d(A x, B y)},\right. \\
\frac{d(A x, S x) d(B y, S x)}{1+d(S x, T y)}, \frac{d(A x, T y) d(B y, T y)}{1+d(S x, T y)}, \\
\frac{d(A x, S x) d(B y, T y)}{1+d(A x, B y)}, \frac{d(S x, B y) d(A x, B y)}{1+d(A x, B y)}, \\
\left.\frac{d(B y, S x) d(S x, T y)}{1+d(S x, T y)}, \frac{d(A x, S x) d(S x, T y)}{1+d(S x, T y)}, \frac{d(S x, T y) d(B y, T y)}{d(A x, B y)+d(S x, T y)}\right\},
\end{array}
$$

reduces to:

$$
\begin{array}{r}
\frac{(1+\iota)|x|^{2}}{36} \precsim q\left\{\frac{(1+\iota)|x|^{2}}{4}, 0, \frac{(1+\iota)|x|^{2}}{40}, \frac{4(1+\iota)^{2}|x|^{4}}{9\left[4+(1+\iota)|x|^{2}\right]}, 0,0, \frac{(1+\iota)^{2}|x|^{4}}{4\left[36+(1+\iota)|x|^{2}\right]},\right. \\
\left.\frac{(1+\iota)^{2}|x|^{4}}{4\left[4+(1+\iota)|x|^{2}\right]}, \frac{4(1+\iota)^{2}|x|^{4}}{9\left[4+(1+\iota)|x|^{2}\right]}, 0\right\} .
\end{array}
$$

This may be valid for at least one $q$, for which $0 \leq q<\frac{1}{s^{2}+s}=\frac{1}{6}$. For instance, $\frac{1}{9} \leq q<\frac{1}{6}$.

Case-3. $x=y=0$. In this case $A x=B y=S x=T y=0$ and all metric values are zero. This, by definition (B2) of 'complex-valued b-metric space', is zero.

Hence, all conditions satisfy, and "zero" is the unique common fixed point of $A, B, S, T$. This validates our main Theorem 3.1.

The following example validates our second main theorem 3.2. 
Example 3.2. Let $(X, d)$ be a complex valued $b$ metric space, where $X=[0,1]$ and $d: X \times X \rightarrow \mathbb{C}$ by: $\quad d(x, y)=(1+\iota)|x-y|^{2}$. Define $A, B, S, T: X \rightarrow X$ by: $A x=\frac{x}{24}$, $B x=\frac{x^{2}}{32}, S x=\frac{x}{3}$ and $T x=\frac{x^{2}}{4}$. Then for all $x, y \in X$, we evaluate

$d(A x, B y)=(1+\iota)|A x-B y|^{2}=\frac{1+\iota}{64}\left|\frac{x}{3}-\frac{y^{2}}{4}\right|^{2}$,

$d(S x, T y)=(1+\iota)|S x-T y|^{2}=(1+\iota)\left|\frac{x}{3}-\frac{y^{2}}{4}\right|^{2}$,

$d(A x, S x)=(1+\iota)|A x-S x|^{2}=\frac{49(1+\iota)}{576}|x|^{2}$,

$d(B y, T y)=(1+\iota)|S x-T y|^{2}=\frac{49(1+\iota)}{1032}|y|^{4}$,

$d(B y, S x)=(1+\iota)|S x-B y|^{2}=(1+\iota)\left|\frac{x}{3}-\frac{y^{2}}{32}\right|^{2}$,

$d(A x, T y)=(1+\iota)|A x-B y|^{2}=\frac{1+\iota}{16}\left|\frac{x^{2}}{6}-y^{2}\right|^{2}$.

Observe that:

If $\left\{z_{n}\right\}=\left\{\frac{1}{n}\right\}$ and $\left\{w_{n}\right\}=\left\{\frac{-1}{n}\right\}$ be two sequences in $X$ then:

$\lim _{n \rightarrow \infty} A z_{n}=\lim _{n \rightarrow \infty} S z_{n}=0 \in B(X) \bigcap T(X)=\mathbb{C}$. Similarly,

$\lim _{n \rightarrow \infty} B w_{n}=\lim _{n \rightarrow \infty} T w_{n}=0 \in A(X) \bigcap S(X)=\mathbb{C}$. Thus $(A, S)$ satisfy the (CLCS) property in $B(X) \bigcap T(X)=\mathbb{C}$, and $(B, T)$ satisfy the same property in $A(X) \bigcap S(X)=\mathbb{C}$. So the condition (i) satisfy. The inequality (ii) will satisfy as in Ex.3.1 of [46].Further, mappings $(A, S)$ and $(B, T)$ are weakly compatible at $x=0$ and $y=0$ respectively. Observe that 0 is the unique common fixed point of $A, B, S, T$. This validates Theorem 3.2 .

\section{REF EREN C ES}

1. A. Aghajani, M. Abbas and J. R. Roshan: Common fixed points of generalized weak contractive mappings in partially ordered b-metric spaces. Math. Slovaka 64 (2) (2014), 941-960. Doi: 10.2478/s12175-014-0250-6.

2. J. Ahmad, A. Azam and S. Saejung: Common fixed point results for contractive mappings in complex valued metric spaces. Fixed Point Theory and Application 2014, (67)(2014).

3. M. Aamri and D. El. Moutawakil: Some new common fixed point theorems under strict contractive conditions. J. Math. Anal. Appl. 27(1) (2002), 181-188.

4. A. Azam, B. Fisher and M. Khan: Common fixed point theorems in complex-valued metric spaces. Numer. Funct. Anal. Optim. 32 (3)(2011), 243-253.

5. S. Bhatt, S. ChoukiYal and R. C. Dimri: Common fixed point of mappings satisfying rational inequality in complex-valued metric spaces. Int. J. Pure Appl. Math. 73(2) (2011), 159-164.

6. S. Bhatt, S. Choukiyal and R. C. Dimri: A common fixed point theorem for weakly compatible maps in complex valued metric spaces. Int. J. Math. Sci. Anal. 1(3) (2011), 1385-1389.

7. S. BANACH: Sür les opérations dans les ensembles abstraits et leur application aux équations intégrales. Fund. Math. 3 (1922), 133-181.

8. I. A. BAKhtin: The contraction principal in quasi-metric spaces. Funct. Anal. 30 (1989), 26-37.

9. S. ChAndok and D. Kumar: Some common fixed point results for rational type contraction mappings in complex valued metric spaces. J. Oper. 2013, Article ID 813707 (2013). 
10. S. Chauhan, W. Sintunavarat and P. Kumam: Common fixed point theorems for weakly compatible mappings in fuzzy metric spaces using (JCLR) property. Applied Mathematics 3(9) (2012), 976-982.

11. S. Chauhan: Fixed points of weakly compatible mappings in fuzzy metric spaces satisfying common limit in the range property. Indian J. Math. 543(3) (2012), 375-397.

12. S. Chauhan, M. A. Khan and S. Kumar: Unified fixed point theorems in fuzzy metric spaces via common limit range property. J. Ineql. Appl. 2013, Article ID 182 (2013).

13. S. Czerwik: Contraction mappings in b-metric spaces. Acta Math. Inform. Univ. Ostraviensis 1 (1993), 5-11.

14. A. K. Dubey, Rita Shukla and R. P. Dubey: Common fixed point theorems in complex valued b metric spaces. Journal of complex system 2015, Article ID 832467, 7 pages.

15. M. Hakwadia, R. K. Gujetia and D. K. Mali: Fixed point theorem in complex valued metric spaces for continuity and complexity. Amer. Int. J. Res. Sci. Tech. Engg. Math. 2014, 217-223.

16. M. ImdAD, S. ChaUhAN and P. KumAm: Fixed point theorems for two hybrid pairs of non-self mappings under joint common limit range property in metric spaces. Journal of nonlinear and convex analysis 16(2) (2015), 243-254.

17. M. Imdad, B. D. Pant and S. Chauhan: Fixed point theorems in Menger space using the $\left(C L R_{S T}\right)$ property and Applications. J. Nonlinear Anal. Optim.- Theory and Applications 3(2) (2012), 225-237.

18. M. JAin, K. TAS, S. Kumar and N. Gupta: Coupled fixed point theorems for a pair of weakly compatible maps along with $C L R_{g}$ property in fuzzy metric spaces. J. Applied Math. 2012, Article ID 961210, 13 pages.

19. G. JungCK: Compatible mappings and common fixed points, Internat. J. Math. Math. Sci. 9(4) (1986), 771-779.

20. G. JUnGCK: Common fixed points for non-continuous non-self mappings on a nonnumeric spaces. Far East J. Math. Sci. 4(2) (1996), 199-212.

21. M. Kumar, P. Kumar and S. Kumar: Common fixed point theorems in complex valued metric spaces. J. Anal. Num. Theory 2(2) (2014), 103-109.

22. M. Kumar, P. Kumar and S. Kumar: Some common fixed point theorems using $\left(C L R_{g}\right)$-property in cone metric spaces. Advances in Fixed Point Theory 2(3) (2012), $340-356$.

23. M. Kumar, P. Kumar, S. Kumar and S. M. Kang: Common fixed points for weakly compatible mappings in complex valued metric spaces, Int. J. Pure Appl. Math. 92(3) (2014), 403-419.

24. S. Manro: Some new common fixed point theorems in complex valued metric space. South Asian J. Math. 5, (1)(2015), 13-24.

25. S. Manro, S. S. Bhatia, S. Kumar, P. Kumam and S. Dalal: Weakly compatible mappings along with CLRS property in Fuzzy metric spaces. J. Nonlinear Anal. Appl. 2013, Article ID 00206, 12 pages.

26. S. K. Mohanta and R. Maitra: Common fixed points for $\phi$-pairs in complex valued metric spaces. Int. J. Math. Comp. Res. 1(9) (2013), 251-256. 
27. A. A. Mukheimer: Some common fixed point theorems in complex valued b-metric spaces. Sci. World. Jour. 2014, Article ID 587825.

28. N. MALhotra and B. BAnsal: Some common coupled fixed point theorems for generalized contraction in b-metric spaces. Jour. Nonlinear Sci. Appl. 8 (2015), 8-16.

29. M. ÖzTüRK: Common fixed point theorems satisfying contractive type conditions in complex valued metric space. Abst. Appl. Anal. 2014, Article ID 598465.

30. M. ÖZTüRK and N. KAPLAN: Common fixed points of $f$-contraction mappings in complex valued metric spaces. Math. Sci. 2014, 8:129(2014).

31. R. P. PANT: Common fixed points of noncommuting mappings. J. Math. Anal. Appl. 188 (1994), 436-440.

32. H. K. Pathak, R. R. Lopéz and R. K. Verma: A common fixed point theorem using implicit relation and property (EA) in metric spaces. Filomat(Niš) 21(2) (2007), $211-234$.

33. H. K. Pathak, Y. J. Сho and S. M. KAnG: Remarks on R-weakly commuting mappings and common fixed point theorems. Bull. Korean Math. Soc. 34(2) (1997), $247-257$.

34. H. K. Pathak, R. R. Lopez and R. K. Verma: A common fixed point theorem of integral type using implicit relation. Nonlinear funct. Anal. Appl. 15(1) (2009), 1-12

35. K. RaO, P. Swami and J. Prasad: A common fixed point theorem in complex valued b-metric spaces. Bull. Math. Stat. Res. 1(1) (2013), 1-8.

36. H. PIRI and H. Asharfi: Some fixed point theorems in complete partial b-metric spaces. Advances in Fixed Point Theory 4(3) (2014), 444-461.

37. A. Roldan, E. KARApinar and P. Kumam: Irremisible stimulate on 'Unified fixed point theorems in fuzzy metric spaces via common limit range property'. J. Ineq. Appl. 2014, 2014:257.

38. A. Roldan and W. Sintunavarat: Common fixed point theorems in fuzzy metric spaces using the $\left(C L R_{g}\right)$ property. Fuzzy Sets and System 282 (2016), 131-142.

39. F. RouZKARD and M. IMDAD: Some common fixed point theorems on complex valued metric spaces, Comp. Math. Appl. 64(6) (2012), 1866-1874.

40. S. Shukla and S. S. PAGeY: Some common fixed point theorems in complex valued metric spaces satisfying (E.A) property and (CLR) property, Int. J. Sci. Inno. Math. Res. 2 (4)(2014), 399-407.

41. Y. R. Sharma: Common fixed point theorems in complex valued metric spaces. Int. J. Inno. Res. Sci. Engg. Tech. 2(12) (2013), 8282-8286.

42. W. Sintunavarat and P. Kumam: Common fixed point theorems for a pair of weakly compatible mappings in fuzzy metric spaces. Journal of Applied Mathematics (2011), Article ID 637958, 14 pages.

43. W. Sintunavarat and P. Kumam: Common fixed points for R-weakly commuting in fuzzy metric spaces. Annali dell'Universita' di Ferrara 58 (2012), 389-406.

44. W. Sintunavarat and P. Kumam: Generalized common fixed point theorems in complex valued metric spaces and applications. J. Inequal. Appl. 2012, 84 (2012).

45. W. Sintunavarat, Y. J. Cho and P. Kumam: Ursyhon integral equations approach by common fixed point theorems in complex valued metric spaces. Advances in Differential equation 49 (2013). 
46. D. Singh, O. P. Chauhan, Naval Singh and Vishal Joshi: Common fixed point theorems in complex valued b-metric spaces. Advances in Fixed Point Theory $\mathbf{5}(2)$ (2015), 263-280.

47. D. Singh, O. P. Chauhan, Nanal Singh and Vishal Joshi: Common fixed point theorems in complex valued b-metric spaces. Advances in Fixed Point Theory 5(3) (2015), 412-429.

48. K. Sitthikul and S. SAejung: Some fixed point theorems in complex valued metric spaces. Fixed Point Theory and Appllications 2012, 189: 2012. doi:10.1186/1687-18122012-189.

49. N. Wairojuana W. Sintunanavarat and P. Kumam: Common tripled fixed points for $W$-compatible mappings along with $C L R_{g}$ property in abstract metric spaces. J. ineq. Appl. 2014, 2014:133.

50. R. K. Verma and H. K. PATHAK: Common fixed point theorems using property (E.A) in complex valued metric spaces. Thai. J. Math. 11(2) (2013), 347-355.

51. X. Q. Hu: Common coupled fixed point theorems for contractive mappings in fuzzy metric spaces. Fixed Point Theory and Applications 2011, Article ID 363716, 14 pages, 2011.

\section{R. K. Verma}

Department of Mathematics

Govt. C.L.C. College, Patan

491111 Distt.-Durg, C.G., India

rohitverma1967@rediffmail.com 